\title{
Relationships between Decreasing Force and Muscle Oxygenation Kinetics during Sustained Static Gripping
}

\author{
Shunsuke Yamaji ${ }^{1)}$, Shinichi Demura ${ }^{2)}$, Yoshinori Nagasawa ${ }^{3)}$ and Masakatsu Nakada ${ }^{4)}$ \\ 1) Fukui National College of Technology \\ 2) Department of Physical Education, Kanazawa University \\ 3) Akita Prefectural University \\ 4) National Defence Academy
}

\begin{abstract}
The purposes of this study were to clarify the kinetics of muscle oxygenation (Oxy-Hb, Deoxy-Hb) by near infrared spectroscopy (NIR) in the decreasing force phase, especially the pre- and post-phases of the inflection point, in sustained maximal static gripping (SSG), and to examine the relationship between kinetics of muscle oxygenation and forcedecreasing parameters. The experiment was conducted on 20 male subjects aged 15-18 years. The time at the lowest Oxy$\mathrm{Hb}$ value $(20.5 \pm 5.5 \mathrm{~s})$ significantly correlated with the decreasing times of forces of 40,60 and $80 \%$ of maximal voluntary contraction (MVC), and the rate of decreasing force for $0-1 \min (r=.60, .53, .49$, and -.63 respectively). These parameters reflect the decreasing force based on the oxygenation deficiency into the muscle with the obstruction of the blood flow. The time of reaching the highest Deoxy-Hb value $(46.8 \pm 15.0 \mathrm{~s})$ and the regression coefficient in the Deoxy-Hb decreasing phase correlated significantly with the decrement for 1-2 min. This parameter evaluates the phase where resumption of the blood flow began, and Deoxy-Hb in the tissue was eliminated. The inflection point of the gripping force is related to the time at the highest Deoxy- $\mathrm{Hb}$, and reflects the beginning and the resumption of the blood flow. The decrement for 2-3 min and the regression coefficient of post-inflection point evaluate the steady state phase of force decreasing, in which oxygen is sufficiently supplied to active muscles. J Physiol Anthropol Appl Human Sci 23 (2): 41-47, $2004 \mathrm{http} / /$ www.jstage.jst.go.jp/browse/jpa
\end{abstract}

Keywords: Muscle endurance, Near infrared spectroscopy, Inflection point

\section{Introduction}

The sustained static grip test is a reliable estimate of muscle endurance (Asmussen, 1968; Royce, 1958; Walamies and Tunjanmaa, 1993). The parameters to evaluate muscle endurance by the sustained force curve are the time to reach a force decrease to a certain level (\%MVC), the rate of force decrease or the average integrated area. However, the findings for muscle endurance obtained from previous studies cannot be simply compared, because the measurement conditions, such as intensity or measurement time, are considerably different. Furthermore, the contribution of physiological factors (e.g., muscle oxygenation, recruitment of muscle fiber, excitationcontraction coupling, energy supply to muscle) involving the sustained maximal static muscle contraction changes over time (Hermansen, et al., 1967; Humphreys and Lind, 1963; Kagaya, 1994; Nielson and Ingvar, 1967). The evaluation or interpretation of muscle endurance in an analysis of the sustained force curve can, therefore, be different depending on the evaluated phase (Kagaya and Iwamura, 1989; Kagaya, 1994).

The force value during SSG decreases markedly during the initial phase, mainly reflecting the fatigue of fast tissue fiber (West et al., 1995; Yamaji et al., 2000; Yamaji et al., 2002). The oxygen transport capacity to the active muscle decreases in the initial phase during the SSG because of the blood flow obstruction caused by an increase in intra-muscular pressure (Kimura et al., 1999). Following the decrease of the gripping force, the resumption of blood flow occurs with a decrease of the intra-muscular pressure (Yamaji et al., 2000, Yamaji et al., 2002). The force value then decreases gradually and reaches an almost steady state. Furthermore, it has been clarified that the mean power spectrum density of an electromyogram (EMG) during SSG shifts to a low frequency band as work continues, i.e. the firing frequency of efferent impulses decreases with muscle fatigue and muscle fiber recruitment mobilizes changes all the time. The contribution of physiological factors related to muscle contraction, such as muscle recruitment or muscle oxygenation kinetics, are dependants on working time at a high-level load close to maximal voluntary contraction.

It is clear that the force decrease during sustained static maximal gripping has two force decrease phases, namely a 
rapid decrease (pre-inflection phase) and an almost steady state (post-inflection phase) (Kagaya and Iwamura, 1989). Kagaya and Iwamura (1989) showed that these two phases appeared in each subject in repeated plantar flexion by subjectively fitting the regression line, suggesting that the inflection point divides the sustained force curve into two phases. They suggested that the inflection point is not only dependent on the change of the decreasing speed of the force, but also on physiological factors, such as muscle fiber recruitment or muscle oxygenation kinetics. However, there are few reports on the time-series relationships between sustained force values and muscle oxygenation kinetics. There are no reports regarding the relationship of a statistically divided curve and muscle oxygenation kinetics of each phase. In the above, many researchers proposed many force-decreasing parameters to assess muscle endurance, and they treated these parameters equally. However, we considered that it is important when assessing muscle endurance to divide these parameters based on the physiological mechanism.

It is certain that the contribution of physiological factors involving the sustained static maximal muscle contraction changes over time although it is difficult to identify a change point accurately. The contribution of physiological differences before and after the changing point (inflection point) of force decreasing during sustained muscle contraction may explain a viewpoint of muscle oxygenation kinetics. Although there have been many studies on measuring oxygenation kinetics during sustained static force exertion (Kahn et al., 1998), few studies have examined the relationships between oxygenation kinetics and sustained muscle contraction, and the correspondence of both parameters is not well known. De Blasi et al. (1993) reported that the oxygen consumption in the forearm in the initial phase (one minute) during sustained static maximal forearm flexion, measured by NIR, was very similar irrespective of the presence or absence of blood flow limitations, indicating that the sustained maximal force exertion restricts muscle blood flow at the initial phase. There are no studies that clarify the duration of the influence by the obstructed blood flow, or that identify the relationship between the muscle oxygenation and sustained muscle contraction after the resumption of the blood flow.

The purposes of this study were to clarify the kinetics of muscle oxygenation by NIR in the decreasing force phase, especially the pre- and post-phases of the inflection point, in SSG, and to examine the relationship between kinetics of muscle oxygenation and force-decreasing parameters.

\section{Methods}

\section{Subjects}

Subjects were 20 males, aged 15-18 years [mean \pm SD height $171.4 \pm 3.7 \mathrm{~cm}$, body mass $63.2 \pm 7.9 \mathrm{~kg}$, maximal grip strength $44.3 \pm 5.2 \mathrm{~kg}$ ]. All subjects were healthy without upper extremity impairments. Their physical characteristics approximated the standard values for Japanese males within that age range (Lab Physical Edu in Tokyo Met Univ, 2000). The written informed consent was obtained from all subjects and their parents after a full explanation of the experimental purpose and protocol.

\section{Materials}

Grip strength was measured using a digital hand dynamometer with a load-cell sensor (EG-290, Sakai, Japan). Each signal during SSG was sampled at $20 \mathrm{~Hz}$ by an analog-todigital interface, and then relayed to a personal computer. To increase the motivation of the subjects during SSG, the recorded digital data was immediately displayed on a screen as a sustained force curve to give feedback.

Near infrared (NIR) spectroscopic measurements was used to evaluate muscle oxygenation by the forearm during SSG. The NIR instrument (PSA-IIIN, Biomedical Science, Japan) consisted of a probe and a computerized control system (Nagashima et al, 2000). The probe contained a light source that was filtered at 700,750 and $830 \mathrm{~nm}$, and two optical detectors were placed $15 \mathrm{~mm}$ and $25 \mathrm{~mm}$, respectively, from the light source. The transmitted light from the probe was then either absorbed or scattered within the tissue. Scattered light was delivered via two fiber-optic light detectors to a photomultiplier at every $0.1 \mathrm{sec}$. The optical path length of the NIR light in tissue was evaluated by the direct method or using the Monte Carlo simulation. The mean depth of measurement in the tissue has been confirmed to be half of the distance between the light source and the detector (Hamaoka et al., 1992). According to this hypothesis, the measured mean depth of PSA-IIIN was approximately 7-12.5 mm. We measured the thickness of subcutaneous fat on the region where the NIR probe was attached (forearm) using an ultrasound B-mode imaging device (EUB-200, Hitachi Medical Corp., Japan) in a prior experiment. Thicknesses of all subjects ranged from 2-4 mm. Therefore, PSA-IIIN measures the oxygenation kinetics of tissue more deeply than the subcutaneous fat layer.

The PSA-IIIN used three-wavelengths and two optical detectors to analyze absorbance of three-wavelengths based on the Lambert-Beer law, and to measure the tissue oxygen saturation $\left(\mathrm{StO}_{2}\right)$ and total tissue hemoglobin (Total $\mathrm{Hb}$ ) (Nagashima et al., 2000). Total absorbance of the light incident by the tissue is explained by the sum of the absorbance by the oxygenated hemoglobin (Oxy-Hb) and deoxygenated hemoglobin (Deoxy-Hb) in the blood, and tissue except the blood. Moreover, it is assumed that the difference of the absorbance of tissue except the blood is very small and can be neglected, because three-wavelengths $(700-830 \mathrm{~nm})$ on an NIR spectroscopic is in a very narrow range (Nagashima et al., 2000). The following equation comes from this assumption, and $\mathrm{StO}_{2}$ is measurable even if the mean optical path length cannot be calculated (Hamaoka et al., 1992; Nagashima et al., 2000; Sakai and Saito, 1995).

$$
\mathrm{StO}_{2}=\mathrm{Ca} \cdot \bar{d} /(\mathrm{Ca}+\mathrm{Cb}) \cdot \bar{d} \cdot 100=\mathrm{Ca} / \mathrm{Hb} \cdot 100
$$


$\mathrm{Ca}$ : mass of $\mathrm{Oxy}-\mathrm{Hb}, \mathrm{Cb}$ : mass of Deoxy-Hb, $\bar{d}$ : mean optical path length, $\mathrm{Hb}$ : Total $\mathrm{Hb}$ volume

Kawasuji et al. (1997) examined the oxygenation kinetics of myocardial blood measured using PSA-IIIN, and confirmed the validity of $\mathrm{StO}_{2}$. Moreover, we examined a change of forearm blood flow measured by NIR (PSA-IIIN) and strain gauge plethysmograph (EC5R, HOKANSON) using the venous occlusion method in the range of $20-60 \%$ MVC (target value) SSG for $60 \mathrm{sec}$ in a preliminary experiment. As indicated in Figure 1, the change of values measured by both of the measurement devices with an increasing target value had a consistently corresponding relation, and we confirmed the validity of the relative change of Total $\mathrm{Hb}$ and $\mathrm{StO}_{2}$ by PSAIIIN. The principle of PSA-IIIN was discussed in detail in previous research (Nagashima et al., 2000; Sakai and Saito, 1995). In this study, we examined the inter-trial reliabilities of muscle oxygenation kinetics (Oxy-Hb, Deoxy-Hb) and the grip force value in the SSG test obtained from 5 of 20 subjects, and they were very good $\quad\left(r_{x y}=0.901-0.978,0.712-0.982\right.$, $0.968-0.979$, respectively).

\section{Experimental procedure}

The dominant hand of each subject was judged using Oldfield's handedness inventory (Oldfield, 1971). Handgrip measurements were performed on subjects seated in an adjustable ergometric chair. All subjects performed the handgrip test with the dominant hand, and the grip width was individually adjusted to achieve a 90-degree angle with the proximal-middle phalanges. The arm, supported by an armrest, was in a sagittal and horizontal position, the forearm being vertical with the hand in a semi-prone position. The subjects performed the maximal grip test twice before the sustained handgrip test, and the higher exertion value was used as the target value of the test. Before the test, the subjects rehearsed sustained maximal gripping. They were instructed not to change the grip and to maintain a natural, straight posture

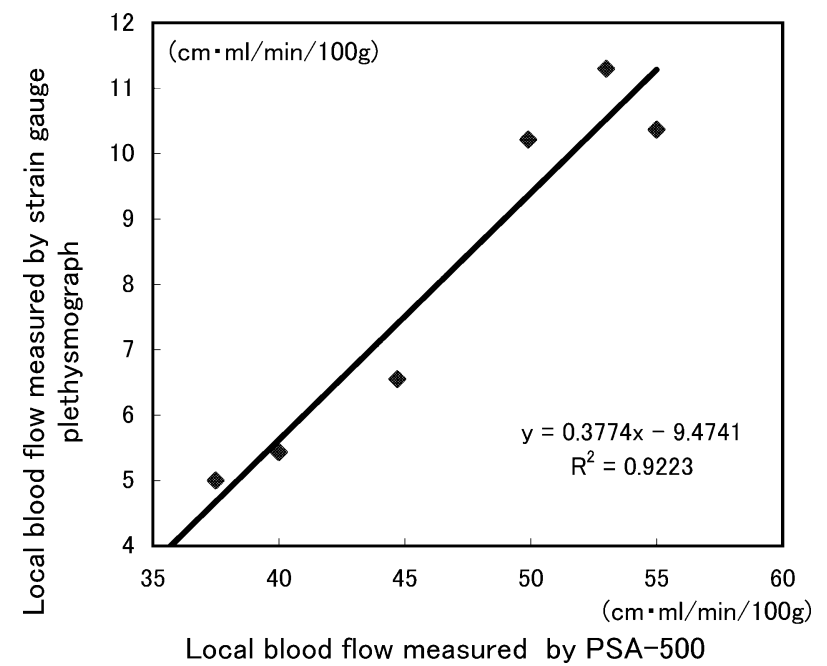

Fig. 1 Relationships of changing local blood flow measured by PSAIIIN and strain gauge plethysmograph during the handgrip measurement. Furthermore, the subjects were instructed to maintain maximal force for $6 \mathrm{~min}$, during which the line of maximal value was displayed on a screen for encouragement. However, no verbal encouragement was given during the test. The NIR probe was positioned over the surface of the flexor digitorum superficialis of the dominant hand, on the ulna side at the tendon of the long palmar muscle, and the muscle oxygenation kinetics was continuously monitored during rest for $2 \mathrm{~min}$ followed by SSG for $6 \mathrm{~min}$. To examine the reproducibility of muscle oxygenation kinetics, 5 of the 20 subjects performed SSG twice for $3 \mathrm{~min}$ with an interval of about one hour between each trial.

\section{Force-decreasing parameters}

Sustained force curves obtained during a SSG test were reproduced with values relative to MVC. We analyzed the property of sustained force curves using the following parameters: time required to decrease from maximal grip strength to $40 \%, 60 \%$, and $80 \% \mathrm{MVC}$; rate of decrease within 0-1 min, 1-2 min and 2-3 min after the onset of gripping; average integrated area during gripping; time at an inflection point, and the regression coefficient at pre-inflection and at post-inflection. The rate of decrease was calculated by averaging the remainders of the subtracted exertion forces from maximal grip strength for each period. The integrated area during gripping was calculated by integrating every value obtained from the relative sustained force curve, and the average integrated area was obtained by dividing the integrated area by the gripping period of $360 \mathrm{~s}$. The inflection point was the time at which the decreasing rate of grip force development markedly changed, and it was calculated statistically from two regression lines fitted to each decreasing phase by applying the two-phase regression model (Kurpad et al., 2001; Lee et al., 1990; Soler et al., 1989). The decrease of the gripping force value in the pre-inflection phase was marked, while in the postinflection phase it was almost at a steady state. The inflection point (time) was determined by the following conditions:

1) The time series sustained force data (360 data) was divided into the former and latter phases at all combinations (e.g. the former: the latter, 3: 357, 4: 356, ., 356: 4, 357: 3), and respective regression lines were calculated (see Figure 2).

2) The best fit regression lines were determined by the following conditions: the regression coefficients (a1) in the pre-inflection phase were significant and greater than the regression coefficients (a2) in any other post-inflection phase, and the sum of the determination coefficients of both regression equations was highest.

3) The inflection point was determined as the time corresponding to the boundary point between the former and latter time series data when combining the best fit regression lines (see Figure 2).

\section{Muscle oxygenation kinetics}

Muscle oxygenation kinetics during SSG, with oxygenated, deoxygenated and total hemoglobin (Oxy-Hb, Deoxy-Hb, and 


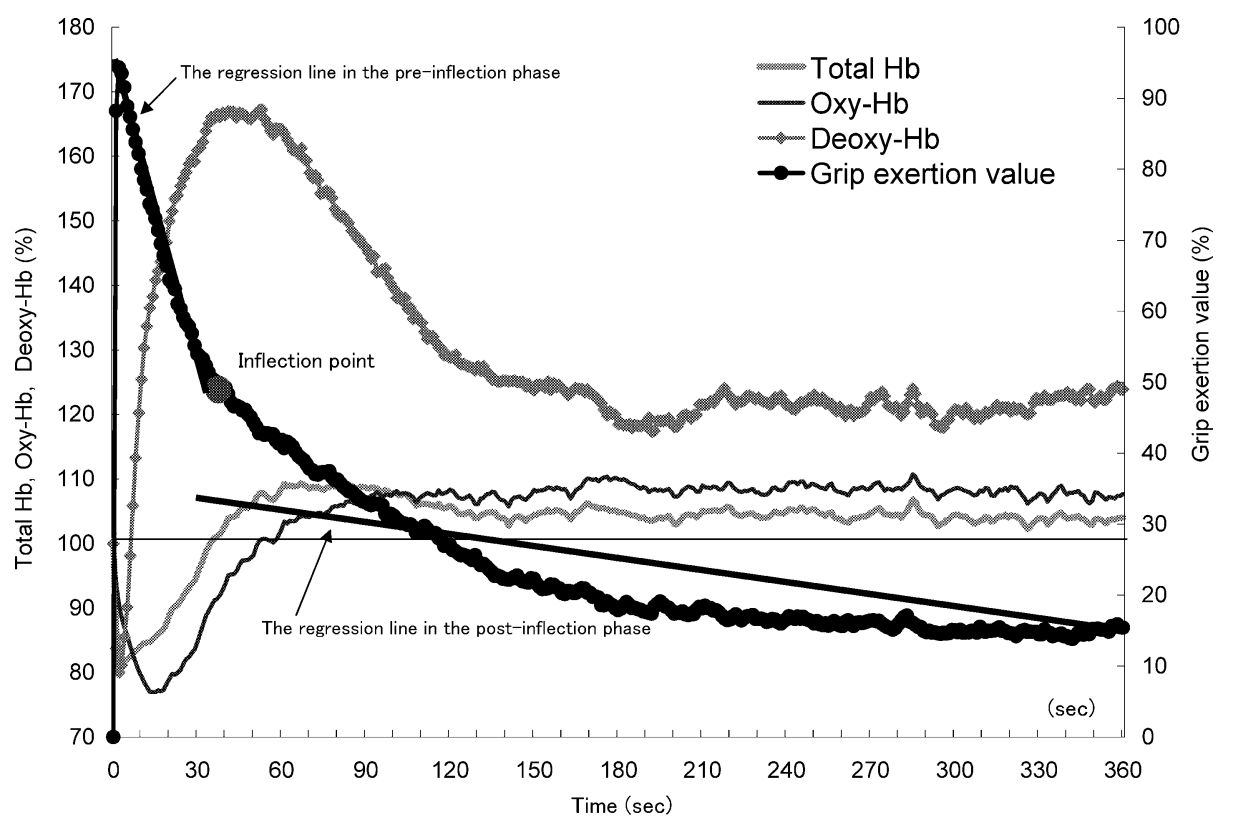

Fig. 2 Average curves of the muscle oxygenation kinetics (Oxy-Hb, Deoxy-Hb) and relative grip force over 6-min during SSG. The discrete data of grip force and oxygenation kinetics are average values calculated from each sampling time $(20 \mathrm{~Hz}$ and $10 \mathrm{~Hz}$, respectively) from each subject's time series data. Two regression lines were calculated using the data set before or after the inflection point, respectively.

Total $\mathrm{Hb}$, respectively) was determined from the following equations;

$$
\begin{aligned}
& \text { Oxy- } \mathrm{Hb}=\text { Total } \mathrm{Hb} \times \text { Oxygen saturation }\left(\mathrm{StO}_{2}\right) \quad(\mathrm{cm} \cdot \mathrm{g} / \mathrm{l}) \\
& \text { Deoxy- } \mathrm{Hb}=\text { Total } \mathrm{Hb}-\mathrm{Oxy}-\mathrm{Hb}
\end{aligned}
$$

Data obtained by NIR spectroscopy was used to calculate the relative value based on resting values for $1 \mathrm{~min}$. The parameters for the change of Oxy-Hb were used for the time of reaching the lowest value during the SSG and the regression coefficient in the markedly increasing phase after reaching the lowest value. The parameters for the change of Deoxy-Hb were used for the time of reaching the highest value during the SSG and the regression coefficient in the markedly decreasing phase after reaching the highest value. The inflection point of the change of Oxy-Hb shifted from the markedly increasing phase after reaching the lowest $\mathrm{Oxy}-\mathrm{Hb}$ value at the steady state phase. The inflection point of the change of Deoxy-Hb shifted from the markedly decreasing phase after reaching the highest Deoxy-Hb value at the steady state phase. Each inflection point was determined by a two-phase regression coefficient when the coefficient of regression estimation and the inflection point of gripping force were at the highest.

\section{Data analysis}

The inter-trial reliabilities of muscle oxygenation kinetics were evaluated by calculating the cross correlation coefficient between two trial values. Pearson's correlation coefficients examined the relationship among all parameters. The probability level of 0.05 was considered indicative of statistical significance.

\section{Results}

Relationships between sustained force curve and muscle oxygenation kinetics

Figure 2 shows the average curves of changes for $\mathrm{Oxy}-\mathrm{Hb}$, Deoxy- $\mathrm{Hb}$, Total $\mathrm{Hb}$ and grip force during sustained static maximal gripping for $6 \mathrm{~min}$. The discrete data for these average curves was used to calculate the average for each sampling time $(20 \mathrm{~Hz}$ and $10 \mathrm{~Hz}$, respectively) of each subject. $\mathrm{Oxy}-\mathrm{Hb}$ tended to decrease over a period of about $20 \mathrm{~s}$ before reaching the lowest gripping value $(20.5 \pm 5.5 \mathrm{~s})$ (Table 1$)$. After this decrease, Oxy-Hb increased for about $80 \mathrm{~s}$ after the onset of gripping, then increased more gradually until about $180 \mathrm{~s}$ after the onset of gripping at which time the changes in $\mathrm{Oxy}-\mathrm{Hb}$ seemed to reach an almost steady state at a higher level compared to that at rest (about 108\%). On the other hand, Deoxy-Hb tended to increase markedly during the first $40 \mathrm{~s}$ after the onset of gripping and reached the highest value $(46.8 \pm 15.0 \mathrm{~s})$. Following this increase, Deoxy-Hb decreased rapidly until about $120 \mathrm{~s}$ after the onset of gripping, then slowly decreased until about $180 \mathrm{~s}$ after the onset of gripping, at which time the changes in Deoxy-Hb seemed to reach an almost steady state with a higher level compared to that at rest (about $120 \%$ ). The lowest Oxy-Hb value was obtained after 10-20 s of gripping, at which time the grip force had decreased to about $60-70 \%$ MVC. The highest Deoxy-Hb value was reached after about $40 \mathrm{~s}$ of gripping, at which time the grip force had decreased to about 50\% MVC. In addition, the grip force after $60 \mathrm{~s}$ of gripping was almost $40 \% \mathrm{MVC}$, and Deoxy- 
Table 1 Correlations between the force-decreasing parameters and muscle oxygenation parameters during SSG

\begin{tabular}{|c|c|c|c|c|c|c|c|c|c|c|c|c|c|c|c|c|}
\hline & & Mean & $\mathrm{SD}$ & (1) & (2) & (3) & (4) & $(5)$ & $(6)$ & (7) & $(8)$ & (9) & $(10)$ & (11) & (12) & (13) \\
\hline \multicolumn{17}{|c|}{ Force-decreasing parameters } \\
\hline (1) & Time to $80 \%(\mathrm{sec})$ & 11.6 & 5.4 & & & & & & & & & & & & & \\
\hline (2) & Time to $60 \%(\mathrm{sec})$ & 25.3 & 11.4 & .83 & & & & & & & & & & & & \\
\hline (3) & Time to $40 \%(\mathrm{sec})$ & 63.5 & 18.4 & .49 & .64 & & & & & & & & & & & \\
\hline (4) & Rate of decrease for $0-1 \min \left(\% \cdot \mathrm{sec}^{-1}\right)$ & 40.4 & 7.8 & -.86 & -.94 & -.77 & & & & & & & & & & \\
\hline (5) & Rate of decrease for $1-2 \min \left(\% \cdot \mathrm{sec}^{-1}\right)$ & 66.5 & 5.6 & & -.46 & -.92 & .62 & & & & & & & & & \\
\hline (6) & Rate of decrease for $2-3 \min \left(\% \cdot \sec ^{-1}\right)$ & 78.2 & 4.5 & & & -.52 & & .69 & & & & & & & & \\
\hline (7) & Average integrated area $\left(\% \cdot \mathrm{sec}^{-1}\right)$ & 27.2 & 3.4 & .56 & .65 & .78 & -.74 & -.82 & -.86 & & & & & & & \\
\hline (8) & Time at an inflection point $(\mathrm{sec})$ & 38.3 & 20.5 & .49 & .77 & & -.64 & & & .53 & & & & & & \\
\hline (9) & $\mathrm{RC}$ of pre-inflection & -1.56 & 0.54 & .61 & .84 & .61 & -.81 & & & .52 & .79 & & & & & \\
\hline$(10)$ & $\mathrm{RC}$ of post-inflection & -0.08 & 0.02 & & & & & .51 & & & & & & & & \\
\hline \multicolumn{17}{|c|}{ Muscle oxygenation parameters } \\
\hline (11) & Time at lowest Oxy-Hb value (sec) & 20.5 & 5.5 & .60 & .53 & .49 & -.63 & & & .50 & & & & & & \\
\hline (12) & $\mathrm{RC}$ in the increasing phase of $\mathrm{Oxy}-\mathrm{Hb}$ & 0.52 & 0.29 & & & & & & & & & & & & & \\
\hline (13) & Time at highest Deoxy-Hb value (sec) & 46.8 & 15.0 & & .59 & .40 & -.51 & & & & .49 & .57 & & & & \\
\hline (14) & $\mathrm{RC}$ in the decreasing phase of Deoxy-Hb & -0.96 & 0.99 & & -.62 & -.61 & .53 & .52 & & -.53 & -.57 & -.57 & & & & -.57 \\
\hline
\end{tabular}

Note: only significant correlations $(\mathrm{p}<.05)$; $\mathrm{RC}=$ regression coefficient.

$\mathrm{Hb}$ began to decrease, whereas $\mathrm{Oxy}-\mathrm{Hb}$ had already returned to the level at rest $(100 \%)$ followed by a further gradual increase. When the grip force almost reached the steady state, changes in $\mathrm{Oxy}-\mathrm{Hb}$ and Deoxy-Hb seemed to be in a steady state.

Relationships between force-decreasing parameters and muscle oxygenation kinetics

The time at the lowest Oxy-Hb value approximately corresponded to the time required for the force to decrease to between 60 and $80 \%$ MVC. A significant correlation was found between the $\mathrm{Oxy}-\mathrm{Hb}$ decrease and the force decrease at $40 \%, 60 \%$ and $80 \% \mathrm{MVC}$, the rate of force decrease for $0-1 \mathrm{~min}$, and the average integrated area during gripping (Table 1). The time at the highest Deoxy-Hb value approximately corresponded to the time required for the force to decrease between $40 \%$ and $60 \% \mathrm{MVC}$, and also to the time of an inflection of the decreasing sustained force curve. The time at the highest Deoxy-Hb value significantly correlated with the time required for the force to decrease between $40 \%$ and $60 \% \mathrm{MVC}$, the rate of force decrease for $0-1 \mathrm{~min}$, the time at an inflection point, and the regression coefficient at preinflection. In addition, the regression coefficient of Deoxy-Hb during the markedly decreasing phase of Deoxy-Hb correlated significantly with the time required for the force to decrease to $40 \%$ and $60 \% \mathrm{MVC}$, the rate of force decrease for $0-1$ and 1-2 min, the average integrated area, the time at an inflection point, and the regression coefficient during the phase of preinflection in the sustained force curve (Table 1).

\section{Discussion and Conclusions}

Muscle blood flow decreases temporarily at the beginning of sustained static muscle contraction at a high level (about 50\%
MVC) (Bonde-Petersen et al., 1975; Gaffney et al., 1990; Kahn et al., 1998; Kimura et al., 1999; Nielson and Ingvar, 1967; Royce, 1958). De Blasi et al. (1993) examined oxygen consumption in the forearm in the initial phase (1 min) during sustained static maximal forearm flexion, measured by NIR, and reported that it was very similar irrespective of the presence or absence of a blood flow limitation, and that sustained static maximal muscle contraction restricts muscle blood flow for about $1 \mathrm{~min}$. It is inferred that the influence of the obstruction of muscle blood flow would continue for about $1 \mathrm{~min}$ after the onset of gripping, because $\mathrm{Oxy}-\mathrm{Hb}$ and Total $\mathrm{Hb}$ were below resting levels for about $50-60 \mathrm{~s}$ in this study. We, therefore, considered that the decrease of $\mathrm{Oxy}-\mathrm{Hb}$ and total $\mathrm{Hb}$ at the initial phase $(20 \mathrm{~s})$ of SSG resulted from the obstruction of the blood flow caused by an increase in intramuscular pressure. In addition, the present study suggests that the gripping at $60-100 \%$ MVC obstructs the blood flow, because the grip force at $20 \mathrm{~s}$ after the onset of the gripping was about $60-70 \% \mathrm{MVC}$. The time to reach the lowest Oxy$\mathrm{Hb}$ value preceded the time to reach the highest Deoxy-Hb value. Deoxy-Hb increased during this period even though $\mathrm{Oxy}-\mathrm{Hb}$ began to increase with the resumption of blood flow because of the high oxygen demand caused by SSG. This disagreement in the change time of $\mathrm{Oxy}-\mathrm{Hb}$ and Deoxy-Hb may be explained by an increase in the oxygen demand with SSG.

It is considered that the parameters, such as the time to decrease to $40 \%, 60 \%$, and $80 \% \mathrm{MVC}$, the decrement for 1 min and the integrated area are related to the markedly decreasing initial phase, since these parameters are correlated with the time to reach the lowest Oxy-Hb and highest Deoxy$\mathrm{Hb}$ values. We, then, inferred that the decrement for $1 \mathrm{~min}$ and the time to decrease until 40\% MVC (about $60 \mathrm{~s}$ ) reflect the decreasing force based on the oxygenation deficiency into the 
muscle with the obstruction of the blood flow. As the correlations between these parameters were high, one or two parameters should be selected from these parameters as reflecting the above stated physiological mechanism.

Royce (1958) compared sustained force curves during sustained static maximal gripping with or without the occlusion of arterial blood flow, and found that the decrease in the grip force was greater at $60 \% \mathrm{MVC}$ with occlusion. This suggests that the decreasing sustained force curve for a maximal contraction depends on blood flow or muscle oxygen kinetics at the phase below $60 \%$ MVC rather than during the initial phase. Deoxy-Hb reached the highest value $(46.8 \pm 15.0 \mathrm{~s})$, and began to decrease at about $60 \mathrm{~s}$ after the onset of gripping, and then became an almost steady state at about $180 \mathrm{~s}$ after the onset of gripping.

We calculated the inflection point in the Deoxy-Hb curve from two regression equations in order to divide the curve into two phases, as well as the point on the Oxy-Hb curve. The inflection point of $\mathrm{Oxy}-\mathrm{Hb}$ (from the lowest value to the steady state phase) and Deoxy-Hb (from the highest value to the steady state phase) curves were obtained at about $100 \mathrm{~s}$ and $160 \mathrm{~s}$ after the onset of gripping, respectively. As shown in the first equation (see Method), Oxy-Hb and Deoxy-Hb do not always change at the same time. Because Oxy-Hb was calculated from $\mathrm{StO}_{2}$ and Total $\mathrm{Hb}$. Therefore, there is a slight time lag between these parameters. This indicates that the $\mathrm{Oxy}-\mathrm{Hb}$ and Deoxy-Hb curves reached an almost steady state after the inflection points. Deoxy-Hb began to decrease when Oxy-Hb returned to $100 \%$, i.e., equal to that at rest, after the onset of gripping. Total $\mathrm{Hb}$ also reached an almost steady state. The changes of Oxy-Hb and Deoxy-Hb after the time of the highest Deoxy-Hb value imply that the Deoxy-Hb was eliminated by a decrease in the obstruction of blood flow, and that the oxygen supply increased by the increased blood flow and the increase of Total $\mathrm{Hb}$ caused by the decrease of exertion force. Total $\mathrm{Hb}$ and $\mathrm{Oxy}-\mathrm{Hb}$ increasing and exceeding $100 \%$ would indicate an increasing resumption of blood flow. Muscle oxygenation kinetics in the phase after reaching the highest Deoxy-Hb value implies that Deoxy-Hb began to be eliminated because of relief of the obstruction of blood flow caused by the resumption of the blood-flow. Thus, the change in this phase indicates that oxygen was satisfactorily supplied to active muscles. That Total $\mathrm{Hb}, \mathrm{Oxy}-\mathrm{Hb}$, and Deoxy-Hb reached an almost steady state at the high level above resting is considered to be an active hyperemia state caused by the sustained gripping. The decrement for 1-2 min correlated significantly to the regression coefficient in the decreasing phase of Deoxy-Hb. It is suggested that this force-decreasing parameter is useful to evaluate the phase that resumption of the blood flow began, and Deoxy-Hb in the tissue was eliminated.

In the present study, time at inflection in the decreasing sustained force curve approximately corresponded with the phase after the time of the highest Deoxy-Hb value. The time at the highest Deoxy-Hb, but not the lowest $\mathrm{Oxy}-\mathrm{Hb}$ value, significantly correlated with the time at inflection in the sustained force curve. The inflection point is a parameter to divide the sustained force curve into two phases, that is, a rapidly decreasing phase for the force (pre-inflection phase) and a gradually decreasing phase (post-inflection phase). The time at the highest Deoxy-Hb value was preceded by the time at the lowest Oxy-Hb value. Thus, the inflection point of the sustained force curve may be related to the time at the highest $\mathrm{Deoxy}-\mathrm{Hb}$ rather than time at the lowest $\mathrm{Oxy}-\mathrm{Hb}$, and the inflection point reflects the beginning the resumption of the blood flow rather than the obstruction of blood flow. It is, therefore, suggested that the inflection point of the decreasing force exertion can divide the sustained force curve into the above-stated phases.

On the other hand, the force exertion value reached an almost steady state in the post-inflection phase (about $150 \mathrm{~s}$ ), in which Deoxy-Hb decreased markedly, followed by a steady state. The marked decrease of Deoxy-Hb occurred after the inflection point. The decrement for $2-3 \mathrm{~min}$ and the regression coefficient of post-inflection point will evaluate the steady state phase of force decreasing, in which oxygen is sufficiently supplied to active muscles. These parameters will evaluate the steady state in both oxygen kinetics in vivo and the performance of muscle contraction. However, as individual differences in force decreased in this phase, further study is needed to examine whether these parameters are as useful as muscle endurance parameters.

The oxygenation kinetics of skeletal muscles in this phase differs from that in the initial phase, showing a marked decrease of the force exertion value. The inflection time of force decreasing is estimated to reflect the muscle oxygenation kinetics. In this study, we used young adult males as subjects, but some researchers have reported that there are sex differences in the sustained force-decreasing curve, especially the latter phase. Therefore, it may be necessary to examine the sex difference in the relationship between the oxygenation kinetics and the force-decreasing curve.

In conclusion, Oxy-Hb decreased for $20.5 \pm 5.5 \mathrm{~s}$ after the onset of SSG, and the gripping force during the time decreased to $60-70 \% \mathrm{MVC}$. The decrement for $1 \mathrm{~min}$ and the time to decrease until 40\% MVC (about $60 \mathrm{~s}$ ) reflect the decreasing force based on the oxygenation deficiency into the muscle with the obstruction of the blood flow. The decrement for 1-2 min will evaluate the phase when the resumption of the blood flow began, and Deoxy-Hb in the tissue was eliminated. The inflection point of the gripping force relates to the time at the highest Deoxy- $\mathrm{Hb}$, and reflects the beginning and resumption of the blood flow. The decrement for 2-3 min and the regression coefficient of the post-inflection point evaluate the steady state phase of force decreasing, in which oxygen is sufficiently supplied to active muscles.

\section{References}

Asmussen E (1968) The neuromuscular system and exercise. In: Falls H, eds. Exercise Physiology. Academic Press, New 
York, 3-42

Barnes WS (1986) The relationship between maximal isometric strength and intramuscular circulatory occlusion. Ergonomics 23: 351-357

Bonde-Petersen F, Mork A, Nielsenm E (1975) Local muscle blood flow and sustained contractions of human arm and back muscles. Eur J Appl Physiol 34: 43-50

De Blasi RA, Cope M, Elwell C, Safoue F, Ferrari M (1993) Noninvasive measurement of human forearm oxygen consumption by near infrared spectroscopy. Eur J Appl Physiol 67: 20-25

Gaffney FA, Sjogaard G, Saltin B (1990) Cardiovascular and metabolic responses to static contraction in man. Acta Physiol Scand 138: 249-258

Hamaoka T, Albani C, Chance B, Iwane H (1992) A new method for the evaluation of muscle aerobic capacity in relation to physical activity measured by near-infrared spectroscopy. Integrat Med Sport Sci 37: 421-429

Hermansen L, Hultman E, Saltin B (1967) Muscle glycogen during prolonged severe exercise. Acta Physiol Scand 71: 129-139

Heyward VH (1975) Influence of static strength and intramuscular occlusion on submaximal static muscle endurance. Res Q Exerc Sport 46: 393-402

Humphreys PW, Lind AR (1963) The blood flow through active and inactive muscle of the forearm during sustained handgrip contraction. J Physiol 166: 120-135

Kagaya A, Iwamura E (1989) Strength decrement in repeated maximal voluntary contractions and muscular endurance performance of plantar flexions. Report of Res Center for Physical Edu 17: 1-17 (in Japanese)

Kagaya A (1994). Muscle endurance - evaluating for physical fitness — . Jpn J Sports Sci 13: 233-240 (in Japanese)

Kahn JF, Jouanin JC, Bussiere JL, Tinet E, Avrillier S, Ollivier JP, Monod H (1998) The isometric force that induces maximal surface muscle deoxygenation. Eur J Appl Physiol, 78: $183-187$

Kawasuji M, Yasuda T, Tomita S, Sakakibara N, Takemura H, Watanabe Y (1997) Near-infrared monitoring of myocardial oxygenation during intermittent warm blood cardioplegia. Eur J Cardio-Thoracic Surgery 12: 236-241

Kimura N, Katsumura T, Hamaoka T, Shimomitsu T (1999) The estimation of oxygen availability in muscle during isometric exercise by near infrared spectroscopy. J Exer Sport Physiol 6: 93-102 (in Japanese)

Kurpad AV, Raj T, El-Khoury A, Beaumier L, Kuriyan R, Srivatsa A, Borgonha S, Selvaraj A, Regan MM, Young VR (2001) Lysine requirements of healthy adult Indian subjects, measured by an indicator amino acid balance technique. Am J Clin Nutr 73: 900-907

Lab Physical Edu in Tokyo Met Univ (2000) New Physical
Fitness Standards of Japanese People, 4th ed. Fumaido, Tokyo, 98-101 (in Japanese)

Lee ML, Poon WY, Kingdon HS (1990) A two-phase linear regression model for biologic half-life data. J Lab Clin Med 115: 745-748

Nagashima Y, Yada Y, Hattori M, Sakai A (2000) Development of a new instrument to measure oxygen saturation and total hemoglobin volume in local skin by near-infrared spectroscopy and its clinical application. Int $\mathrm{J}$ Biometeorol 44: 11-19

Nielson B, Ingvar D (1967) Intramuscular pressure and contractile strength related to muscle blood flow in man. Scand J Clinical Lab Inv 99: 31-38

Oldfield RC (1971) The assessment and analysis of handedness: the Edinburgh inventory. Neuropsychologia 9: 97-113

Royce J (1958) Isometric fatigue curve in human muscle with normal and occluded circulation. Res Q Exerc Sport 29: 204-212

Sakai A, Saito T (1995) Noninvasive measurement of tissue oxygen consumption in human using near-infrared spectroscopy. Therapeutic Res 16: 247-250 (in Japanese)

Soler AM, Folledo M, Martins LE, Lima-Filho EC, Gallo Junior L (1989) Anaerobic threshold estimation by statistical modelling. Braz J Med and Biol Res 22: 795-797

Walamies M, Tunjanmaa V (1993) Assessment of the reproducibility of strength and endurance handgrip parameters using a digital analyser. Eur J Appl Physiol 67: $83-86$

West W, Hicks A, Clements L, Dowling J (1995) The relationship between voluntary electromyogram, endurance time and intensity of effort in isometric handgrip exercise. Eur J Appl Physiol 71: 301-305

Yamaji S, Demura S, Nagasawa Y, Nakada M, Yoshimura Y, Matsuzawa Z, Toyoshima Y (2000) Examination of the parameters of static muscle endurance on sustained static maximal hand gripping. Jpn J Physical Edu 45: 695-706 (in Japanese)

Yamaji S, Demura S, Nagasawa Y, Nakada M, Kitabayashi T (2002) The effect of measurement time when evaluating static muscle endurance during sustained static maximal gripping. Appl Human Sci 21: 151-158

Received: May 25, 2003

Accepted: January 15, 2004

Correspondence and reprint requests to: Shunsuke Yamaji, Fukui National College of Technology, Geshi-cho, Sabae-shi, Fukui 916-8507, Japan

Phone: +81-778-62-8229

Fax: +81-778-62-3417

e-mail: yamaji@fukui-nct.ac.jp 